\title{
On the Spectrum and Preconditioning of Electromagnetic Volume Integral Equations
}

\section{Markkanen, Johannes}

IEEE

2016

Markkanen , J , Ylä-Oijala , P \& Järvenpää , S 2016 , On the Spectrum and Preconditioning of Electromagnetic Volume Integral Equations . in 2016 URSI INTERNATIONAL

SYMPOSIUM ON ELECTROMAGNETIC THEORY (EMTS) . URSI International Symposium on Electromagnetic Theory, IEEE , New York , pp. 834-837, URSI International Symposium

on Electromagnetic Theory , Espoo, Finland , 14/08/2016 . https://doi.org/10.1109/URSI-EMTS.2016.7571533

http://hdl.handle.net/10138/228803

https://doi.org/10.1109/URSI-EMTS.2016.7571533

submittedVersion

Downloaded from Helda, University of Helsinki institutional repository.

This is an electronic reprint of the original article.

This reprint may differ from the original in pagination and typographic detail.

Please cite the original version. 


\title{
On the Spectrum and Preconditioning of Electromagnetic Volume Integral Equations
}

\author{
Johannes Markkanen*, Pasi Ylä-Oijala ${ }^{\dagger}$, and Seppo Järvenpää ${ }^{\dagger}$ \\ * Department of Physics, University of Helsinki, Finland \\ e-mail: johannes.markkanen@helsinki.fi \\ $\dagger$ Department of Radio Science and Engineering, Aalto University, Finland \\ e-mails: pasi.yla-oijala@aalto.fi, seppo.jarvenpaa@aalto.fi
}

\begin{abstract}
Spectral properties of current-based volume integral equation of electromagnetic scattering are investigated in the case of isotropic and bi-isotropic objects. Using Helmholtz decomposition the spectrum is derived separately for the solenoidal, irrotational, and harmonic subspaces. Based on this analysis, preconditioning strategies of the matrix equation are discussed.
\end{abstract}

\section{INTRODUCTION}

Volume integral equation (VIE) methods are useful in many electromagnetic scattering analysis tasks involving inhomogeneous dielectric and magnetic targets. Since the method requires only the Green's function of the background medium, that most often is homogeneous and isotropic, the method is available for much more complicated media than e.g., the surface integral equation method requiring Green's functions of all considered media.

The challenge in VIE is that it results a dense fully populated matrix that is expensive to solve. Hence, fast solvers like the ones based on Fast Fourier Transform or on Fast Multipole Method, are mandatory as the size of the problem is increased. Since these fast methods are based on the iterative solution of the matrix equation, for the total efficiency of the method it is essential that the iterative solution converges as fast as possible.

Spectrum of electromagnetic VIEs has been studied e.g., in [1]-[4]. These studies show that the spectral radius depends on the permittivity, or on the material contrast in general. The spectral radius in turn is related to the conditioning of the matrix and to the convergence rate of an iterative solution. As verified with numerical experiments, strong material contrast and high frequency, have a negative effect on the iteration count [5]. Even more problematic are structures with negative material parameters. For these cases the spectrum of the VIE operator appears on both size of the origin and the matrix can be singular.

As shown in [6] in the case of dielectric isotropic medium an efficient preconditioner for the electric current VIE operator can be obtained by properly scaling the solenoidal, irrotational and harmonic subspaces. This preconditioning strategy leads to an iteration count that is nearly independent on the permittivity, as long as the permittivity is positive.

In this paper, we extend the analysis of the current-based VIE from isotropic media to bi-isotropic one. In the latter case, the spectrum has a more complicated dependence on the material parameters and several problematic material parameter combinations are identified that may lead to a singular matrix. Preconditioning strategies based on the discrete Helmholtz decomposition are also discussed.

\section{Current-Based Volume Integral Equation FORMULATION}

Consider time-harmonic electromagnetic scattering (the time factor is $e^{-i \omega t}$ ) by a three-dimensional bounded object $D$ in a homogeneous and isotropic background medium with constant $\varepsilon_{0}$ and $\mu_{0}$. The sources of the incident field $\mathbf{E}^{\mathrm{inc}}, \mathbf{H}^{\mathrm{inc}}$ are outside the object.

A solution to the scattering problem is found by using the volume integral equation (VIE) method. This method is based on the volume equivalence principle where the total electromagnetic fields, scattered plus incident, are expressed as

$$
\begin{aligned}
\mathbf{E}(\mathbf{r}) & =\frac{-1}{i \omega \varepsilon_{0}} \mathcal{L}[\mathbf{J}](\mathbf{r})-\mathcal{K}[\mathbf{M}](\mathbf{r})+\mathbf{E}^{\mathrm{inc}}(\mathbf{r}) \\
\mathbf{H}(\mathbf{r}) & =\frac{-1}{i \omega \mu_{0}} \mathcal{L}[\mathbf{M}](\mathbf{r})+\mathcal{K}[\mathbf{J}](\mathbf{r})+\mathbf{H}^{\mathrm{inc}}(\mathbf{r})
\end{aligned}
$$

Here the volume integral operators are

$$
\begin{aligned}
& \mathcal{K}[\mathbf{F}](\mathbf{r})=\nabla \times \mathcal{V}[\mathbf{F}](\mathbf{r}), \\
& \mathcal{L}[\mathbf{F}](\mathbf{r})=\left(\nabla \nabla \cdot+k_{0}^{2}\right) \mathcal{V}[\mathbf{F}](\mathbf{r}),
\end{aligned}
$$

and $\mathcal{V}$ is given by

$$
\mathcal{V}[\mathbf{F}](\mathbf{r})=\int_{D} G_{0}\left(\mathbf{r}, \mathbf{r}^{\prime}\right) \mathbf{F}\left(\mathbf{r}^{\prime}\right) d V^{\prime},
$$

with the Green's function $G_{0}$ of the background medium. Definition of the equivalent electric and magnetic volume current densities $\mathbf{J}$ and $\mathbf{M}$ depend on the medium. In the following we consider two cases, isotropic and bi-isotropic medium. 


\section{A. Isotropic Object}

Assume first that object $D$ is isotropic with position dependent scalar parameters $\varepsilon_{1}$ and $\mu_{1}$. Then the constitutive relations are given by

$$
\left[\begin{array}{l}
\mathbf{D} \\
\mathbf{B}
\end{array}\right]=\left[\begin{array}{cc}
\varepsilon_{0} \varepsilon_{r} \overline{\overline{\mathcal{I}}} & \overline{\overline{\mathcal{O}}} \\
\overline{\overline{\mathcal{O}}} & \mu_{0} \mu_{r} \overline{\overline{\mathcal{I}}}
\end{array}\right]\left[\begin{array}{l}
\mathbf{E} \\
\mathbf{H}
\end{array}\right]
$$

where $\overline{\overline{\mathcal{I}}}$ and $\overline{\overline{\mathcal{O}}}$ is the unity and zero dyadic and $\varepsilon_{r}$ and $\mu_{r}$ are the relative electric permittivity and magnetic permeability of the medium.

The equivalent electric and magnetic volume current densities are now given by

$$
\begin{aligned}
\mathbf{J} & =-i \omega \varepsilon_{0} \tau_{\varepsilon} \mathbf{E} \\
\mathbf{M} & =-i \omega \mu_{0} \tau_{\mu} \mathbf{H},
\end{aligned}
$$

with notations $\tau_{\varepsilon}=\varepsilon_{r}-1$ and $\tau_{\mu}=\mu_{r}-1$.

Using (1) and (2) with (7) and (8), leads to the electric and magnetic current volume integral equation (JMVIE)

$$
\left[\begin{array}{cc}
\mathcal{I}-\tau_{\varepsilon} \mathcal{L} & -i \omega \varepsilon_{0} \tau_{\varepsilon} \mathcal{K} \\
i \omega \mu_{0} \tau_{\mu} \mathcal{K} & \mathcal{I}-\tau_{\mu} \mathcal{L}
\end{array}\right]\left[\begin{array}{c}
\mathbf{J} \\
\mathbf{M}
\end{array}\right]=\left[\begin{array}{c}
\mathbf{J}^{\mathrm{inc}} \\
\mathbf{M}^{\mathrm{inc}}
\end{array}\right]
$$

Here we have denoted

$$
\mathbf{J}^{\mathrm{inc}}=-i \omega \varepsilon_{0} \tau_{\varepsilon} \mathbf{E}^{\mathrm{inc}} \text { and } \mathbf{M}^{\mathrm{inc}}=-i \omega \mu_{0} \tau_{\mu} \mathbf{H}^{\mathrm{inc}} .
$$

\section{B. Bi-isotropic Object}

For bi-isotropic medium the constitutive relations contain also the relative magneto-electric parameters $\xi_{r}$ and $\zeta_{r}$ [7]

$$
\left[\begin{array}{l}
\mathbf{D} \\
\mathbf{B}
\end{array}\right]=\left[\begin{array}{cc}
\varepsilon_{0} \varepsilon_{r} \overline{\overline{\mathcal{I}}} & \sqrt{\varepsilon_{0} \mu_{0}} \xi_{r} \overline{\overline{\mathcal{I}}} \\
\sqrt{\varepsilon_{0} \mu_{0}} \zeta_{r} \overline{\overline{\mathcal{I}}} & \mu_{0} \mu_{r} \overline{\overline{\mathcal{I}}}
\end{array}\right]\left[\begin{array}{l}
\mathbf{E} \\
\mathbf{H}
\end{array}\right] .
$$

Parameters $\xi_{r}$ and $\zeta_{r}$ can be related to Tellegen $\chi_{r}$ and chiral $\kappa_{r}$ parameters as [7]

$$
\xi_{r}=\chi_{r}-i \kappa_{r} \quad \text { and } \quad \zeta_{r}=\chi_{r}+i \kappa_{r}
$$

The volume currents in bi-isotropic medium are given by

$$
\begin{aligned}
\mathbf{J} & =-i \omega \varepsilon_{0} \tau_{\varepsilon} \mathbf{E}-i \omega \xi \mathbf{H}, \\
\mathbf{M} & =-i \omega \mu_{0} \tau_{\mu} \mathbf{H}-i \omega \zeta \mathbf{E} .
\end{aligned}
$$

Using representations (1) and (2) with (13) and (14), gives JMVIE for bi-isotropic medium

$$
\begin{gathered}
{\left[\begin{array}{cc}
\mathcal{I}-\tau_{\varepsilon} \mathcal{L}+i \omega \xi \mathcal{K} & -i \omega \varepsilon_{0} \tau_{\varepsilon} \mathcal{K}-\frac{\xi_{r}}{\eta_{0}} \mathcal{L} \\
i \omega \mu_{0} \tau_{\mu} \mathcal{K}-\zeta_{r} \eta_{0} \mathcal{L} & \mathcal{I}-\tau_{\mu} \mathcal{L}-i \omega \zeta \mathcal{K}
\end{array}\right]\left[\begin{array}{c}
\mathbf{J} \\
\mathbf{M}
\end{array}\right]} \\
=\left[\begin{array}{c}
\mathbf{J}^{\text {inc }} \\
\mathbf{M}^{\text {inc }}
\end{array}\right] .
\end{gathered}
$$

Here $\eta_{0}=\sqrt{\mu_{0} / \varepsilon_{0}}$ and

$$
\begin{aligned}
\mathbf{J}^{\mathrm{inc}} & =-i \omega \varepsilon_{0} \tau_{\varepsilon} \mathbf{E}^{\mathrm{inc}}-i \omega \xi \mathbf{H}^{\mathrm{inc}} \\
\mathbf{M}^{\mathrm{inc}} & =-i \omega \mu_{0} \tau_{\mu} \mathbf{H}^{\mathrm{inc}}-i \omega \zeta \mathbf{E}^{\mathrm{inc}}
\end{aligned}
$$

\section{SPECTRAL ANALYSIS}

Next we study the spectrum of JMVIE formulation in the case of isotropic and bi-isotropic medium. First we note that JMVIE defines a mapping from $L^{2}(D)$ onto itself [6], [8]. In the following, we shall utilize Helmholtz decomposition of $L^{2}(D)$

$$
L^{2}(D)=\nabla H_{0}^{1}(D) \oplus \mathcal{H}_{0}\left(\operatorname{div}_{0}, D\right) \oplus \mathcal{W}(\partial D),
$$

where $\nabla H_{0}^{1}(D)$ is the irrotational subspace (with zero curl), $\mathcal{H}_{0}\left(\operatorname{div}_{0}, D\right)$ is the solenoidal subspace (with zero divergence), and $\mathcal{W}(\partial D)$ is the subspace of the gradients of harmonic $H^{1}(D)$ fields.

Operator $\mathcal{K}$ is compact in $L^{2}(D)$ and its eigenvalues accumulate to the origin. Therefore, it suffices to consider the $\mathcal{L}$ operator. To analyze that operator on the irrotational subspace, we shall use identity

$$
\mathcal{L}[\mathbf{F}]=(\mathcal{T}-\mathcal{I})[\mathbf{F}]
$$

with $\mathcal{T}[\mathbf{F}]=\nabla \times(\nabla \times \mathcal{V})[\mathbf{F}]$.

\section{A. Isotropic Medium}

Consider first the solenoidal part in the isotropic medium. Since for a solenoidal function $\mathbf{F}^{\mathrm{sol}}, \mathcal{L}\left[\mathbf{F}^{\mathrm{sol}}\right]=k_{0}^{2} \mathcal{V}\left[\mathbf{F}^{\mathrm{sol}}\right]$, and $\mathcal{V}$ is compact, the essential spectrum of that part is simply the unity.

To analyze the irrotational part we apply (19) to obtain

$$
\mathcal{I}-\tau_{\varepsilon} \mathcal{L}=\varepsilon_{r} \mathcal{I}-\tau_{\varepsilon} \mathcal{T}
$$

Since for an irrotational function $\mathbf{F}^{\text {irr }}$ operators $\mathcal{T}$ and $\mathcal{K}$ vanish, the integral operator of (9) on irrotational subspace reduces to

$$
\left[\begin{array}{cc}
\varepsilon_{r} \mathcal{I} & 0 \\
0 & \mu_{r} \mathcal{I}
\end{array}\right]
$$

The essential spectrum (accumulation points of the eigenvalues) for the irrotational subspace is thus given by

$$
\lambda=\varepsilon_{r} \quad \text { and } \quad \lambda=\mu_{r} .
$$

Finally, consider the harmonic subspace. Writing $\mathbf{J}=\mathbf{J}^{\text {harm }}=$ $\nabla h$ and substituting that current into the $\mathcal{L}$ operator gives after integration by parts [6]

$$
\mathcal{L}\left[\mathbf{J}^{\text {harm }}\right]=\left(\mathcal{I}+\tau_{\varepsilon}\left(\nabla \mathcal{S}_{n}-k^{2} \mathcal{V}\right)\right)\left[\mathbf{J}^{\text {harm }}\right]
$$

Here

$$
\mathcal{S}_{n}[\mathbf{F}](\mathbf{r}):=\int_{\partial D} G_{0}\left(\mathbf{r}, \mathbf{r}^{\prime}\right) \mathbf{n}\left(\mathbf{r}^{\prime}\right) \cdot \mathbf{F}\left(\mathbf{r}^{\prime}\right) d S^{\prime}
$$

is the surface single layer potential operator. Taking normal component of the right hand side of (23) on the surface of $D$ gives

$$
\left(\mathbf{n} \cdot \mathcal{I}+\tau_{\varepsilon}\left(\mathcal{D}_{n}^{*}-k^{2} \mathbf{n} \cdot \mathcal{V}\right)\right)\left[\mathbf{J}^{\text {harm }}\right]
$$

where $\mathcal{D}_{n}^{*}$ is the adjoint of the surface double layer operator. Since that operator is compact if the surface is smooth, the essential spectrum of the harmonic part has accumulation points at $\left(\varepsilon_{r}+1\right) / 2$ and $\left(\mu_{r}+1\right) / 2$. For non-smooth surfaces, 
the adjoint of the double layer potential operator is bounded and the spectrum will be spread around the accumulation points.

In conclusion, in isotropic medium the essential spectrum of JMVIE (9) contains the following accumulation points

$$
1,\left(1+\varepsilon_{r}\right) / 2,\left(1+\mu_{r}\right) / 2, \varepsilon_{r} \text { and } \mu_{r} .
$$

\section{B. Bi-isotropic Medium}

Next we consider bi-isotropic medium. The main difference compared to the isotropic medium is that the $\mathcal{L}$ operator appears on both the diagonal and off-diagonal blocks of (15).

Consider first the solenoidal subspace. Since the $\mathcal{L}$ operator is compact for solenoidal functions, the essential spectrum of the solenoidal part is unity, as in the case of isotropic medium.

For the irrotational part we utilize (20), and identity (19) is used to obtain

$$
\xi \mathcal{L}=\xi \mathcal{I}-\xi \mathcal{T}
$$

Then, the integral operators in (15) reduce to

$$
\left[\begin{array}{ll}
\varepsilon_{r} \mathcal{I} & \xi_{r} \mathcal{I} \\
\zeta_{r} \mathcal{I} & \mu_{r} \mathcal{I}
\end{array}\right]
$$

The spectrum is therefore determined by

$$
\operatorname{det}\left[\begin{array}{cc}
\left(\varepsilon_{r}-\lambda\right) \mathcal{I} & \xi_{r} \mathcal{I} \\
\zeta_{r} \mathcal{I} & \left(\mu_{r}-\lambda\right) \mathcal{I}
\end{array}\right]=0
$$

This leads to a quadratic equation which solution is

$$
\lambda=\frac{\varepsilon_{r}+\mu_{r} \pm \sqrt{\left(\varepsilon_{r}+\mu_{r}\right)^{2}-4\left(\varepsilon_{r} \mu_{r}-\xi_{r} \zeta_{r}\right)}}{2}
$$

defining the accumulation points of the spectrum of the irrotational subspace.

Using similar procedure as above in the case of isotropic medium for the harmonic subspace we obtain the following equation (compact operators are again omitted)

$$
\left[\begin{array}{cc}
\frac{1}{2}\left(\varepsilon_{r}+1\right) \mathcal{I} & \frac{1}{2} \xi_{r} \mathcal{I} \\
\frac{1}{2} \zeta_{r} \mathcal{I} & \frac{1}{2}\left(\varepsilon_{r}+1\right) \mu_{r} \mathcal{I}
\end{array}\right]
$$

Solving that equation, gives the following eigenvalues

$$
\begin{gathered}
\lambda=\frac{1}{4}\left(\epsilon_{r}+\mu_{r}+2\right) \\
\pm \frac{\sqrt{\frac{1}{4}\left(\epsilon_{r}+\mu_{r}+2\right)^{2}-\left(\left(\epsilon_{r}+1\right)\left(\mu_{r}+1\right)-\xi_{r} \zeta_{r}\right)}}{2} .
\end{gathered}
$$

In conclusion, in bi-isotropic media the essential spectrum of (15) consists of unity (solenoidal subspace), (30) (irrotational) and (32) (harmonic).

\section{NUMERICAL EXPERIMENTS}

In the numerical solution, first the volume of an objects is divided into linear tetrahedra. Then the integral equations are discretized using Galerkin's method with piece-wise constant basis and testing functions scaled with the square of the volume of an element. More details on the discretization process are given, e.g., in [5], [6], [9].

Let us consider a sphere with $k_{0} r=0.1$ where $k_{0}$ is the wavenumber of vacuum and $r$ is the radius of the sphere. Fig. 1 shows the eigenvalues for isotropic material with $\varepsilon_{r}=3, \varepsilon_{r}=$ $9, \mu_{r}=1$ and with zero magneto-electric parameters. In the figure, the accumulation points of the spectrum predicted by the theory are denoted for the solenoidal part by a vertical blue line, for the irrotational one with green lines, and for the harmonic part with black lines.

For $\varepsilon_{r}=3$ the spectrum has accumulation points close to 1 and 3 , corresponding to the solenoidal and irrotational subspaces, and the spectrum of the harmonic part is spread around the accumulation point 2 as a consequence of nonsmooth discretization. As permittivity is increased, the shape of the spectrum remains about the same, but the irrotational and harmonic parts become wider. This means that the spectral radius depends on the permittivity. The higher permittivity, the wider is the spectral radius.

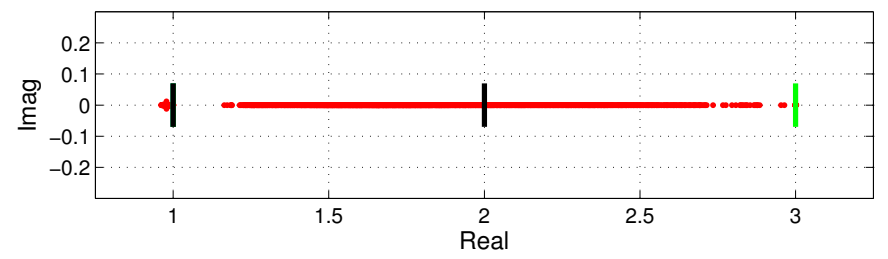

(a) $\varepsilon_{r}=3$

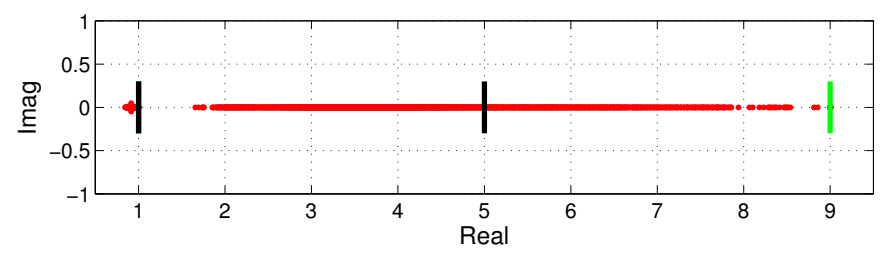

(b) $\varepsilon_{r}=9$

Fig. 1: Eigenvalues (red dots) for a sphere $k_{0} r=0.1$ with $\varepsilon_{r}=3, \varepsilon_{r}=9$ and $\mu_{r}=1, \chi_{r}=0, \kappa=0$.

Next the analysis is repeated with non-zero chiral parameter $\kappa$. As $\kappa$ is increased, Fig. 2, the solenoidal part stays at 1 , the irrotational part starts to shift to lower and higher values, and the harmonic part is slightly wider than in the case of isotropic medium.

As a last example, we consider two problematic cases where the spectrum is spread on both sides of the origin, causing an ill-conditioned matrix and poor iteration convergence. In the first case $\varepsilon_{r}=3, \mu_{r}=1, \chi_{r}=0, \kappa=2$ and in the second one $\varepsilon_{r}=-2, \mu_{r}=1, \chi_{r}=0, \kappa=0.3$. As Fig. 3 illustrates, for these material parameter combinations the accumulation 


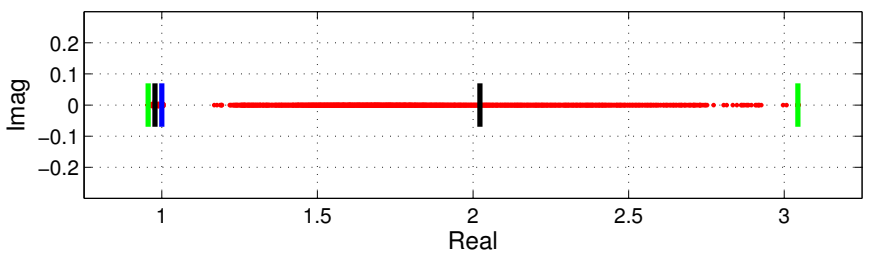

(a) $\kappa=0.3$

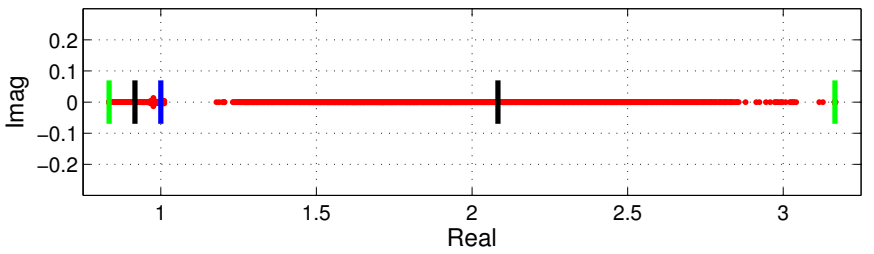

(b) $\kappa=0.6$

Fig. 2: Eigenvalues for a sphere $k_{0} r=0.1$ with $\varepsilon_{r}=3, \mu_{r}=$ $1, \chi_{r}=0$ and with $\kappa=0.3$, and $\kappa=0.6$.

points of the irrotational and harmonic parts appear on the both sides of the origin.

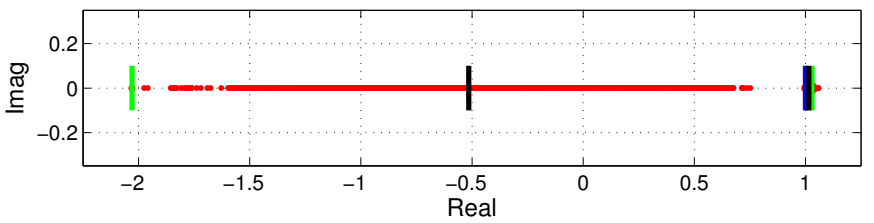

(a) $\varepsilon_{r}=3, \kappa=2$

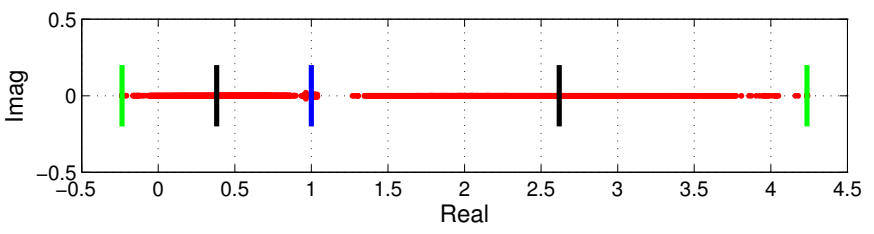

(b) $\varepsilon_{r}=-2, \kappa=0.3$

Fig. 3: Eigenvalues for a sphere $k_{0} r=0.1$ with $\varepsilon_{r}=3, \mu_{r}=$ $1, \chi_{r}=0, \kappa=2$ and with $\varepsilon_{r}=-2, \mu_{r}=1, \chi_{r}=0, \kappa=0.3$.

\section{Preconditioning Strategy}

Next we discuss how the spectral analysis can be utilized in developing a preconditioner for JMVIE. In order to do that we should be able to scale the solenoidal, irrotational and harmonic parts separately [6]. This in turn requires the use of a discrete Helmholtz decomposition. To that end, let $\mathcal{P}^{S}, \mathcal{P}^{I}$ and $\mathcal{P}^{H}$ denote the projections from the piece-wise constant $L^{2}$ space to the solenoidal, irrotational and harmonic subspaces. Let

$$
\mathbf{A} \mathbf{x}=\mathbf{b}
$$

denote the original matrix equation due to the discretization of JMVIE in the case of an isotropic dielectric scatterer. With the Helmholtz projection operators, the scaled equation can be expressed as [6]

$$
\mathcal{P} \mathbf{A}(\mathcal{H} \mathbf{x})=\mathcal{P} \mathbf{b}
$$

where $\mathcal{H}=\mathcal{P}^{S}+\mathcal{P}^{I}+\mathcal{P}^{H}$ is the total Helmholtz projector and the preconditioner is given by

$$
\mathcal{P}=\mathcal{P}^{S}+\frac{1}{\varepsilon_{r}} \mathcal{P}^{I}+\frac{2}{1+\varepsilon_{r}} \mathcal{P}^{H} .
$$

As shown in [6], this preconditioner leads to an iteration count that is nearly independent on the permittivity of the object.

\section{CONCLUSION}

Spectral properties of the current-based volume integral equation are studied in the case of isotropic and bi-isotropic media. Using Helmholtz decomposition the spectrum is divided into solenoidal, irrotational, and harmonic parts. For electrically small objects, the solenoidal and irrotational parts of the discretized system follow rather well the theory and have a discrete spectrum. Due to non-smooth discretizations the harmonic part is spread over the accumulation point. The main point in this spectral analysis is that it can be utilized in developing efficient preconditioners for the discrete matrix equations.

\section{ACKNOWLEDGMENT}

This work was supported by the Academy of Finland, grant 259950 entitled Computational and Mathematical Models for Electromagnetic Wave Interaction with Complex Material Structures (COMMA), and by the ERC Advanced Grant No. 320773 entitled Scattering and Absorption of Electromagnetic Waves in Particulate Media (SAEMPL).

\section{REFERENCES}

[1] N.V. Budko and A.B. Samokhin, "Spectrum of the volume integral operator of electromagnetic scattering", SIAM Journal on Scientific Computing, vol. 28(2), pp. 682-700, 2006

[2] M. Costabel, E. Darrigrand, and E Koné, "Volume and surface integral equations for electromagnetic scattering by a dielectric body", Journal of Computational and Applied Mathematics, vol. 234(6), pp. 1817-1825, 2010.

[3] M. Costabel, E. Darrigrand, and H. Sakly, "The essential spectrum of the volume integral operator in electromagnetic scattering by a homogeneous body", Comptes Rendus Mathematique, vol. 350(3), pp. 193-197, 2012.

[4] J. Markkanen and P. Ylä-Oijala, "Numerical comparison of spectral properties of volume-integral-equation formulations, Journal of Quantitative Spectroscopy and Radiative Transfer, in press.

[5] J. Markkanen, P. Ylä-Oijala, and A. Sihvola, "Discretization of the volume integral equation formulations for extremely anisotropic materials", IEEE Trans. Antennas Propag., vol. 60, pp. 5195-5202, 2012.

[6] J. Markkanen, Discrete Helmholtz decomposition for electric current volume integral equation formulation, IEEE Trans. Antennas Propag. vol. 62(12), pp. 6282-6289, 2014.

[7] A. Sihvola, Electromagnetic Mixing Formulas and Applications, IEE Electromagnetic Wave Series 47, Hertfordshire, United Kingdom, IEE, 1999.

[8] M.C. van Beurden and S.J.L. van Eijndhoven, "Well-posedness of domain integral equations for a dielectric object in homogeneous background", J. Eng. Math, vol. 62, pp. 289-302, 2008.

[9] S. Järvenpää, J. Markkanen, and P. Ylä-Oijala, "Broadband multilevel fast multipole algorithm for electric-magnetic current volume integral equation", IEEE Trans. Antennas Propag., vol. 61(8), pp. 4393-4397, 2013. 